\title{
Surgical repair of a large intrathoracic meningocele associated with neurofibromatosis type 1 after failed cystoperitoneal shunts: illustrative case
}

\author{
Christopher H. F. Sum, MBBS, ${ }^{1}$ Lai-Fung Li, FRCSEd(SN), ${ }^{1}$ Benedict B. T. Taw, FRCSEd(SN), ${ }^{1}$ Wai-Man Lui, FRCSEd, ${ }^{1}$ \\ Ko-Yung Sit, FRCSEd, ${ }^{2}$ Velda L. Y. Chow, FCSHK, ${ }^{3}$ and Yat-Wa Wong, FRCSEd ${ }^{4}$ \\ Divisions of ${ }^{1}$ Neurosurgery, ${ }^{2}$ Cardiothoracic Surgery, and ${ }^{3}$ Plastic and Reconstructive Surgery, Department of Surgery, and ${ }^{4}$ Department of Orthopedics and Traumatology, \\ Faculty of Medicine, The University of Hong Kong, Hong Kong, China
}

\begin{abstract}
BACKGROUND Surgical treatment of intrathoracic meningoceles, commonly associated with neurofibromatosis type 1 (NF1), aims to reduce sac size for symptomatic relief. The procedures can be divided into cerebrospinal fluid diversion and definitive repair. The authors describe the management of an intrathoracic meningocele in a 56-year-old female with preexisting NF1.

OBSERVATIONS The patient presented with progressive dyspnea. Magnetic resonance imaging revealed a left hemithoracic meningocele arising from the thecal sac at C7-T2. Two attempts at diversion by cystoperitoneal shunts resulted in recurrence. For definitive repair, T2-3

costotransversectomy was performed, and intradural closure of the meningocele opening was performed utilizing spinal dura and autologous fascia lata graft. Trapezius muscle regional flap was turned for reinforcement. Persistent leak warranted reoperation 7 days later. A transthoracic approach was undertaken using video-assisted thoracoscopic resection of the sac at aortic arch level, with reinforcement by latissimus dorsi flap and synthetic materials. Mechanical pleurodesis was performed. Intradural repair of the meningocele opening was revised.
\end{abstract}

LESSONS Inherent dural abnormality makes repair difficult for meningoceles associated with NF1. A combined intradural and thoracoscopic approach with regional muscle flap and synthetic material reinforcement is a unique method for definitive treatment. Some essential points of perioperative management are highlighted.

https://thejns.org/doi/abs/10.3171/CASE21404

KEYWORDS neurofibromatosis type 1; intrathoracic meningocele; intradural; transthoracic; VATS

Neurofibromatosis type 1 (NF1) is an autosomal dominant neurocutaneous disorder with dysplasia of the skeletal and nervous systems. Dural ectasia is a downstream effect due to inherent dural weakness. This may manifest as intrathoracic meningoceles, which are characterized by saccular protrusion of the dura and arachnoid mater through a pathologically dilated thoracic intervertebral foramen or vertebral defect. This rare condition occurs as part of the constellation of NF1 in $60-85 \%$ of cases. ${ }^{1-3}$ Additional associations occur with Marfan syndrome, achondroplasia, and ankylosing spondylitis, as well as iatrogenic trauma postlaminectomy. ${ }^{4,5}$

We present a patient with a large intrathoracic meningocele associated with NF1, in whom two consecutive shunting procedures failed.
Two operations were subsequently performed, which comprised both an intradural and extradural transthoracic approach for definitive treatment.

\section{Illustrative Case}

\section{Presentation and Diagnosis}

A 46-year-old woman had known NF1 with café-au-lait macules, intertriginous freckling, and cutaneous neurofibromas. She presented in 2010 with progressive dyspnea. Plain computed tomography (CT) of the thorax showed an $18.3 \times 9.9 \times 12.5 \mathrm{~cm}$ (longitudinal $\times$ transverse $\times$ anteroposterior) hemithoracic radiopacity with mediastinal shift and tracheal deviation (Fig. 1A and C). Magnetic resonance imaging (MRI) showed a left intrathoracic

ABBREVIATIONS CSF = cerebrospinal fluid; CT = computed tomography; MRI = magnetic resonance imaging; NF1 = neurofibromatosis type 1; VATS = video-assisted thoracoscopic surgery.

INCLUDE WHEN CITING Published September 6, 2021; DOI: 10.3171/CASE21404.

SUBMITTED July 11, 2021. ACCEPTED July 20, 2021.

(c) 2021 The authors, CC BY-NC-ND 4.0 (http://creativecommons.org/licenses/by-nc-nd/4.0/). 


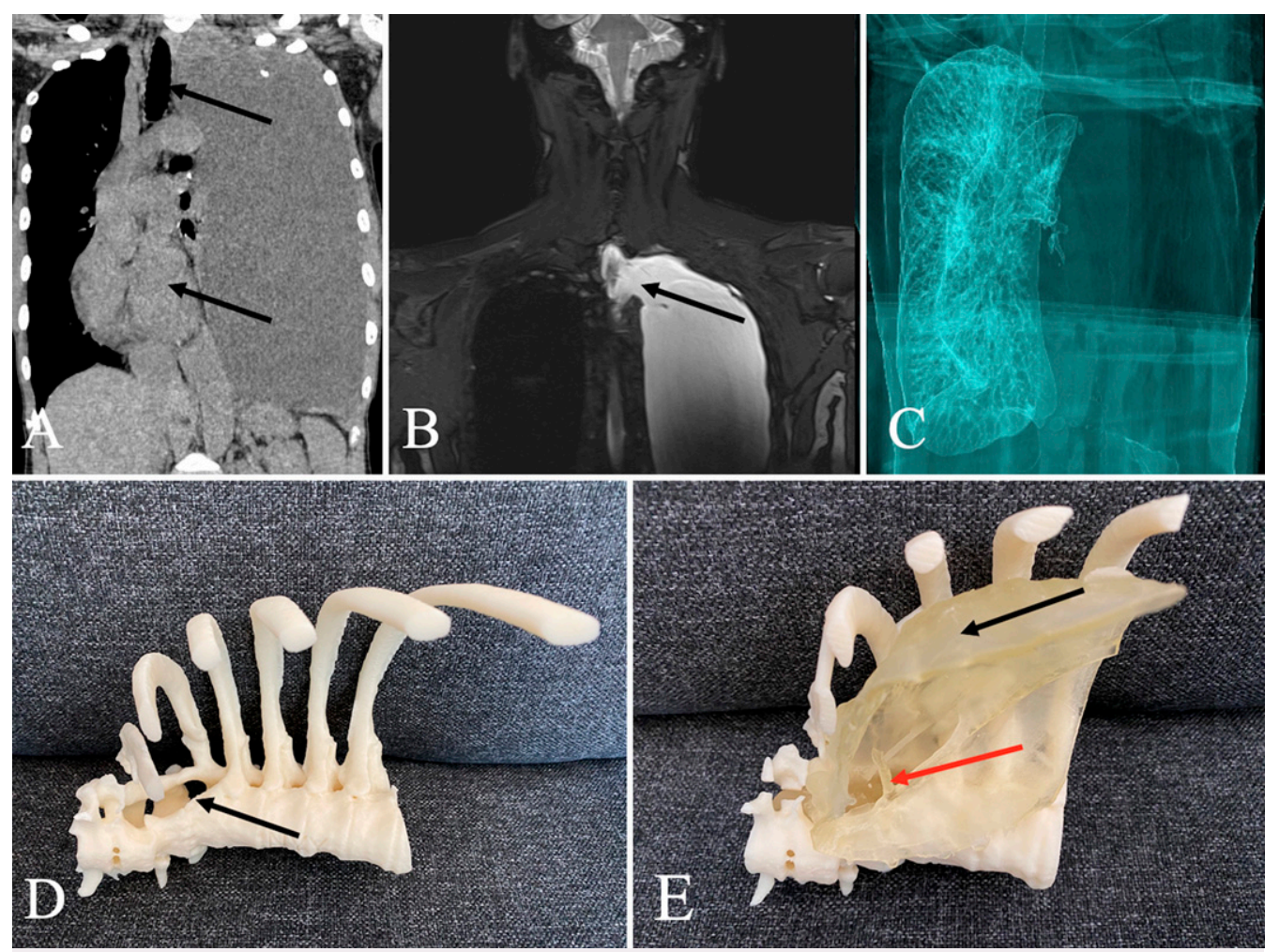

FIG. 1. A: Coronal plain CT scan showing a large left hemithoracic radioopacity with gross tracheal deviation and mediastinal shift (arrows). B: Coronal T2-weighted MRI scan showing a hyperintense cystic lesion with communication (arrow) with the thecal sac. C: Three-dimensional reconstruction highlighting the extent of the thoracic meningocele. D and E: Three dimensional-printed models illustrating the vertebral body defect (arrow) (D) through which the meningocele (black arrow) (E) herniated. E: A T1 nerve root is shown transversing across the meningocele opening (red arrow).

mass with homogenous fluid signal, with communication with the thecal sac (Fig. 1B). The diagnosis was an intrathoracic meningocele. Lung function testing revealed a restrictive pattern, and the forced vital capacity was $41 \%$ of the age-adjusted normal.

\section{Cerebrospinal Fluid Diversionary Procedures}

She opted for a less invasive option, and a cystoperitoneal shunt was inserted in 2011. However, there was reaccumulation within the first week. CT of the thorax showed catheter extrusion. Shunt revision was done in 2014, after which her dyspnea was partially relieved. However, her symptoms worsened again in 2020, with increased mediastinal shift.

\section{First Attempt at Definitive Repair}

A joint-discipline operation was performed in December 2020. A total of $1.4 \mathrm{~L}$ of cerebrospinal fluid (CSF) was tapped from the shunt reservoir in the 2 days prior to surgery. Motor-evoked potential, somatosensory evoked potential, and triggered electromyography were set up for intraoperative monitoring. Endotracheal intubation was performed with a left endobronchial blocker to permit single-lung ventilation. The patient was positioned prone, and using a midline incision, left T2-3 costotransversectomy was made to access the bony defect (Fig. 1D). Via an intradural approach, two $\mathrm{T} 1$ nerve roots (Figs. $1 \mathrm{E}$ and $2 \mathrm{~A}$ ) transversing the meningocele opening were confirmed by stimulation and preserved.

Dissection of the sac from the parietal pleura was abandoned because of arterial bleeding and small operating window. The neck was closed by autologous fascia lata graft (Fig. 2B) using interrupted sutures. This was fostered by a combination of DuraGen (Integra LifeSciences), TachoSil (Baxter), and a left trapezius muscle regional flap. An external ventricular drain was inserted.

\section{Second Attempt at Definitive Repair \\ Transthoracic Part}

Serial postoperative chest radiographs revealed recurrent CSF leak into the meningocele (Fig. 3A-C). The second surgery was performed 7 days later. The patient was placed in a right lateral position, and the cardiothoracic surgeons first resected the sac at the level of aortic arch (Fig. 4A) via a video-assisted thoracoscopic surgery (VATS) approach (Video 1). The residual sac was sutured and reinforced with surgical adhesives. The plastic surgeons harvested the left latissimus dorsi (LD) flap, and it was transposed into the thoracic cavity through an enlarged port site (Fig. 4B). The LD flap reinforced the meningocele repair. Mechanical pleurodesis was performed, and one chest tube was inserted.

VIDEO 1. Clip showing the transthoracic technique via VATS is highlighted. See the main text for a detailed description. Click here to view.

Intradural Part

The patient was then repositioned in the prone position, to reperform the repair of the meningocele opening (Video 2). A multilayered construct was fashioned, with DuraGen placed intradurally, 

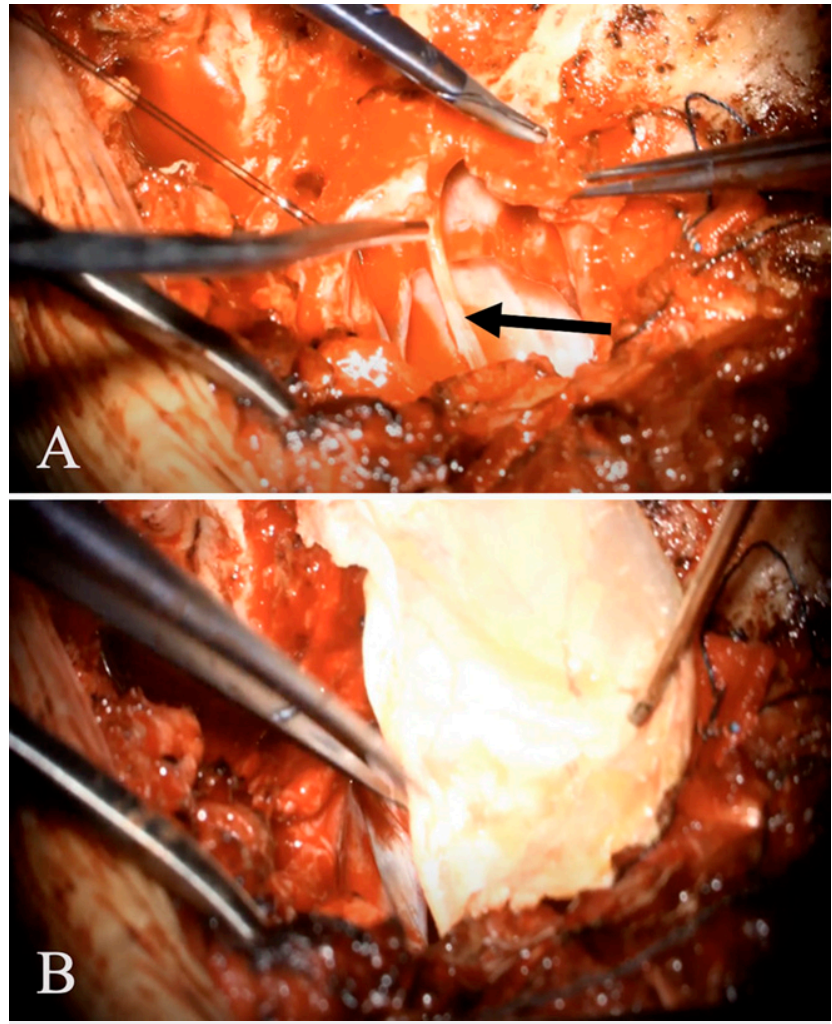

FIG. 2. Intraoperative images. A: T1 nerve root (arrow) shown transversing across the meningocele opening. B: An autologous fascia lata graft was placed intradurally to facilitate the repair.

followed by the fascia lata graft that was resutured with caution to avoid injury to the T1 nerve roots. This was followed by DuraGen, TachoSil, and a Gore-Tex soft-tissue patch placed in an onlay manner. The trapezius muscle flap was repositioned for reinforcing the repair. Valsalva maneuver demonstrated no visible CSF leakage, and the operation was concluded.
VIDEO 2. Clip showing the intradural space was reentered. The fascia lata graft was taken down, and a multilayered construct was created. Click here to view.

Postoperatively, there was good left lung expansion and improvement in the mediastinal shift on chest radiograph (Fig. 5A). CT of the thorax 5 months after surgery showed marked reduction in the meningocele volume (Fig. 5B). The left lung was largely reexpanded, and the mediastinum returned to central. There was complete resolution of her respiratory symptoms.

\section{Discussion}

\section{Observations}

The diagnosis of a thoracic meningocele can be obtained with MRI demonstrating thecal origin of the sac, accompanied by CSF signal within its cavity. CT myelograms can demonstrate the presence of contrast flowing through the subarachnoid space into the meningocele but is usually superfluous. There are often associated osseous abnormalities, such as posterior scalloping of vertebral bodies, kyphoscoliosis, pressure erosion of the pedicles, rib penciling, and transverse process spindling. ${ }^{6-9}$

The postulated pathomechanism is the inability of the weakened, dysplastic dura in withstanding the pulsatile pressure of CSF in response to the cardiac cycle and respiration. ${ }^{10}$ Negative intrathoracic force during inspiration increases the pressure gradient, leading to progressive thecal evagination through intervertebral foramina. Secondary pressure erosion of adjacent bony elements creates a larger osseous orifice, and hence a vicious cycle is formed favoring sac expansion. The usual occurrence of thoracic meningoceles at the apex of the kyphoscoliotic curve solidifies this theory. ${ }^{11}$ Symptoms usually occur at substantial size, as in our patient. Respiratory or neurological symptoms may supervene, like paraparesis, back pain, dyspnea, cough, and palpitations. ${ }^{12}$

Described techniques of surgical treatment are broadly divided into three categories: (a) CSF diversionary procedures, namely cystoperitoneal or lumboperitoneal shunts; (b) intradural approach for meningocele neck repair; and (c) extradural approach for resection or plication
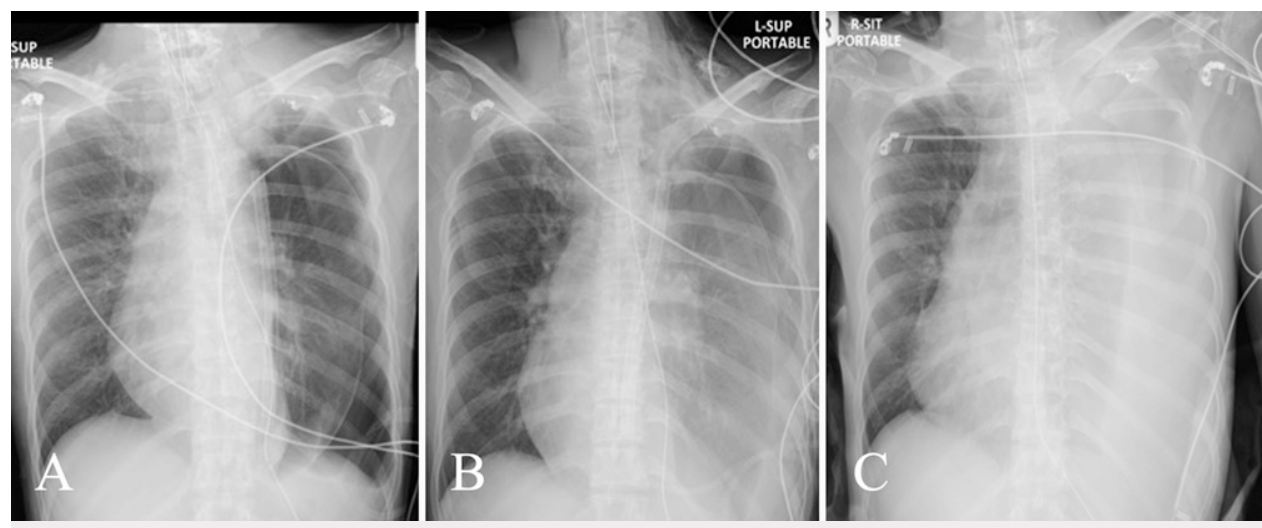

FIG. 3. A-C: Serial chest radiographs (postoperative days 1, 2, and 6, respectively) showing reaccumulation of the meningocele, suggestive of persistent leakage at the intradural repair site. L-SUP = left-supine; R-SIT: right-sitting. 

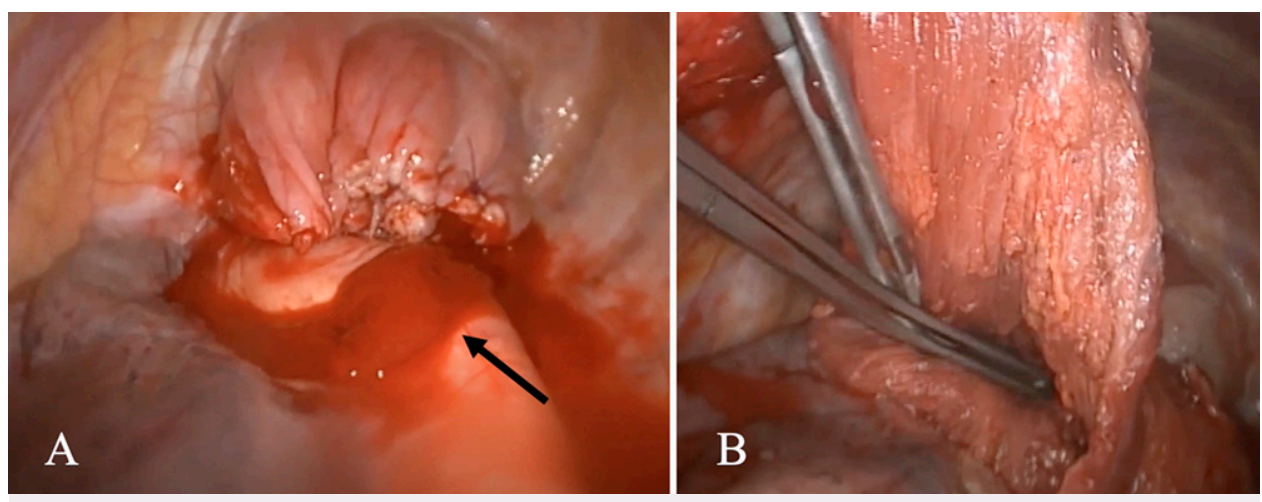

FIG. 4. A: Thoracoscopic view showing the meningocele was resected at the level of the aortic arch (arrow). B: Left LD flap was transposed into the thoracic cavity via a minithoracotomy formed from an enlarged VATS port site.
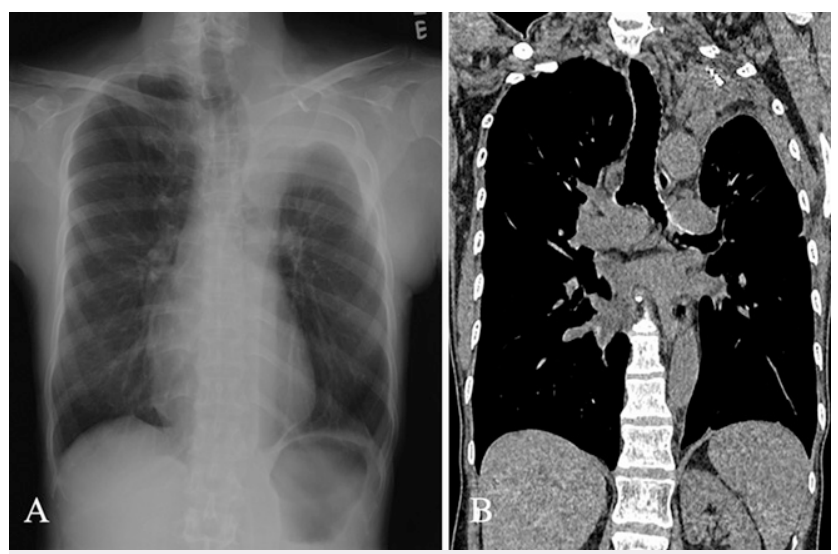

FIG. 5. There was no recurrence shown on the 3-month postoperative chest radiograph (A) or the 5-month postoperative coronal CT scan (B).

of meningocele. Posterior laminectomies, costotransversectomies, or the transthoracic method by thoracotomy or thoracoscopy are variably used for exposure. The ultimate determinants are meningocele size, spinal stability, and available surgical expertise.

Diversionary procedures represent a less invasive strategy; they are quick to perform, and placement under local anesthesia is possible, $^{13}$ a unique solution for candidates with poor respiratory reserve and prohibitive general anesthetic risk. However, as illustrated in our case, shunt failures may occur with catheter extrusion, blockage, or malposition. ${ }^{1}$

Definitive surgeries can be performed intra- or extradurally. An intradural procedure aims to halt the flow of CSF into the sac. The advantage is that nerve roots can be protected, and chest drains can be avoided in nonthoracotomy exposures. However, water-tight dural closure from within the sac can be difficult, due to inherent dural weakness and limited operating window in a deep and narrow space. The possibility of intervening nerve roots can add to the difficulty, as was encountered in our case. Finally, the bulk of meningocele must be left behind in an isolated intradural procedure.

An extradural approach allows plication or resection of the meningocele sac. Small meningoceles can be exposed via posterior laminectomies alone, and moderate-sized ones can be exposed via costotransversectomies, while a thoracoscopic or thoracotomy exposure for large meningoceles is desired, as it provides a wider operative field for dissection. The expense of a large exposure is the need for chest drains and the risk of intrathoracic CSF fistula formation. Laminectomy or costotransversectomy for exposure may compromise stability of the kyphoscoliotic spine, necessitating posterior fusion with or without anterior stabilization.

\section{Lessons}

In the first repair, the access to the neck was limited and hindered by the T1 nerve roots, in which a water-tight closure with fascia graft was not possible. It is debatable whether a pure extradural procedure would suffice, without venturing into the intradural space for neck closure. With both the extradural and intradural procedures, a double fail-safe mechanism is possible. The intradural part theoretically helps reduce the flow of the fistula, should the suture line of the sac fail. The concomitant mechanical pleurodesis performed in the second operation provided a mechanical barrier for the meningocele sac, reducing mass effect on the lungs should recurrence occur. This technique is infrequently reported in the literature. ${ }^{14}$

Messages have also been derived from the perioperative management. Preoperative tapping helped to reduce the acuteness of fluid shift after durotomies and repair. Without preemptive drainage, hypovolemic shock and reexpansion pulmonary edema have been reported. ${ }^{15}$ The most common complication of meningocele surgeries is the formation of meningopleural fistula. ${ }^{3}$ Chest drains are often not placed on suction for the fear of potentiating a fistula formation. Reducing suction duration and force makes theoretical sense and facilitates dural healing.

Large intrathoracic meningoceles are rare pathologies, and the optimal intra- and perioperative management is not well defined. We have revisited its formation mechanism, presentation, diagnosis, the choice of surgical approach, and intraoperative and perioperative management. We share our experience on using a combined intradural and thoracoscopic approach for definitive treatment of a large hemithoracic meningocele.

\section{References}

1. Das P, Goyal T, Hunt MA. Intrathoracic meningocele associated with neurofibromatosis type 1 and a novel technique for surgical repair: case report. J Neurosurg Spine. 2017;27(3):291-294.

2. de Andrade GC, Braga OP, Hisatugo MK-I, de Paiva Neto MA, Succi E, Braga FM. Giant intrathoracic meningoceles associated 
with cutaneous neurofibromatosis type I: case report. Arq Neuropsiquiatr. 2003;61(3A):677-681.

3. Vanhauwaert DJ, Deruytter MJ. Cystoperitoneal shunt as alternative treatment for a giant thoracic meningocele in a patient with neurofibromatosis. Surg Neurol. 2008;69(5):535-537.

4. Fattori R, Nienaber CA, Descovich B, et al. Importance of dural ectasia in phenotypic assessment of Marfan's syndrome. Lancet. 1999;354(9182):910-913.

5. Toyoda K, Taguchi T, Kaneko K, Kato Y, Imajo Y, Imagama T. Highgrade L5 spondylolisthesis associated with dural ectasia in neurofibromatosis. J Orthop Sci. 2005;10(2):233-236.

6. Geerts Y, Marchau M. Intrathoracic meningocele. J Spinal Disord. 1992;5(1):116-121.

7. Malla HP, Park BJ, Koh JS, Jo DJ. Giant intrathoracic meningocele and breast cancer in a neurofibromatosis type I patient. $J$ Korean Neurosurg Soc. 2016;59(6):650-654.

8. Lykissas MG, Schorry EK, Crawford AH, Gaines S, Rieley M, Jain VV. Does the presence of dystrophic features in patients with type 1 neurofibromatosis and spinal deformities increase the risk of surgery? Spine (Phila Pa 1976). 2013;38(18):1595-1601.

9. Sunna TP, Westwick HJ, Zairi F, Berania I, Shedid D. Successful management of a giant anterior sacral meningocele with an endoscopic cutting stapler: case report. J Neurosurg Spine. 2016;24(5):862-866.

10. Yeh Y-C, Lin Y-J, Yeh C-H, Hsieh P-S, Wu C-T. Anterior sacral meningocele repair assisted by intraoperative intrathecal fluorescence and 3D printing model: illustrative case. J Neurosurg Case Lessons. 2021;1(20).

11. Sonoda Y, Tominaga T, Koshu K, Ogawa A, Yoshimoto T. Posterolateral extradural approach for lateral thoracic meningocele-case report. Neurol Med Chir (Tokyo). 1994;34(9):624-627.

12. Rao ZX, Li J, Hang SQ, You C. Congenital spinal intradural arachnoid cyst associated with intrathoracic meningocele in a child. J Zhejiang Univ Sci B. 2010;11(6):429-432.
13. Tanaka K, Shimizu K, Kakegawa S, Oshima K, Takeyoshi I. Cystoperitoneal shunt for a giant intrathoracic meningocele under local anesthesia. Ann Thorac Surg. 2011;91(1):317-319.

14. Yusuf AS, Pillai A, Menon SK, Panikar D. Massive spontaneous hemothorax, giant intrathoracic meningocele, and kyphoscoliosis in neurofibromatosis type 1. J Surg Tech Case Rep. 2014;6(1):33-36.

15. Su YS, Shao B, Pechet TTV, Welch WC. Thoracic meningoceles in NF1: case report and considerations for surgical management. Ann Surg Case Rep. 2019;2(2):1019.

\section{Disclosures}

The authors report no conflict of interest concerning the materials or methods used in this study or the findings specified in this paper.

\section{Author Contributions}

Conception and design: Li, Sum, Taw, Lui, Chow, Wong. Acquisition of data: Li, Sum, Lui, Sit, Chow. Analysis and interpretation of data: Li, Sum, Chow, Wong. Drafting the article: Sum, Lui, Chow. Critically revising the article: Li, Taw, Lui, Chow, Wong. Reviewed submitted version of manuscript: Li, Sum, Taw, Lui, Chow. Approved the final version of the manuscript on behalf of all authors: Li. Statistical analysis: Chow. Administrative/technical/material support: Li, Taw, Lui, Sit, Chow, Wong. Study supervision: Li, Taw, Lui, Chow.

\section{Supplemental Information \\ Videos \\ Video 1. https://vimeo.com/580320797 \\ Video 2. https://vimeo.com/580321681}

\section{Correspondence}

Lai-Fung Li: The University of Hong Kong, Hong Kong, China. Iffrandom@gmail.com. 